\title{
L'espace vécu chez le peuple Hébreu (à l'époque du retour de l'exil. Vlème siècle avant J.-C.)
}

La manière d'appréhender l'espace - de le décrire, d'agir sur lui, d'interpréter la relation qu'on entretient avec lui - varie avec les régions et les époques. Dans le cadre de ce thème général, les pages qui suivent analysent un cas vieux de 25 siècles: celui du peuple hébreu au retour de sa déportation à Babylone. Pourquoi ce choix? Nous disposons, grâce à la Bible, d'une source documentaire écrite, remontant loin, et d'une richesse telle qu'on s'étonne qu'elle ait étē, sous cet angle précis de la perception spatiale, encore aussi peu exploitée.

La mentalité des descendants d'Israël ayant passablement évolué depuis la période des Patriarches jusqu'à l'occupation romaine, une observation portant sur une longue durée conduit à des téléscopages anachroniques. La coupe s'impose donc.

Quant à la fin de siècle retenue ( -538 à - 500 avt. J-C.), elle représente un temps dans la conscience que prend alors la population juive de son attachement au sol. L'expérience récente d'exil et de rapatriement, vécue par un bon quart de la population, n'y est évidemment pas ètrangère.

En schématisant un peu, mais sans solliciter les faits, on peut distinguer trois aspects, de spécificité croissante, et liēs, chacun, à une cause principale différente.

UNE LECTURE CONCENTRIQUE DE L'ORGANISATION DE L'ESPACE.

Thèmes et silences.

Entendue, ici, dans un sens simplement descriptif, la lecture de l'espace comporte un thème majeur et, par référence à des cas plus proches de nous, des demi-mots ou des silences.

La vision omphalique des Hébreux procède d'un chauvinisme élémentaire et vigoureux. Mais banal. Quel peuple y échappe?

L'idée-force est que la terre de Canaan, la Palestine, se situe, moralement, au centre du monde. Alentour, se dispose l'anneau des "nations" avec lesquelles elle est aux prises en quasi-permanence: bédouins du sud-est (Moabites, Edomites, etc...). Egyptiens, Philistins, Phé- niciens, Assyriens, Babyloniens. A leur égard, elle éprouve un sentiment mélangé de supériorité et de crainte obsidionale. (cf. les "oracles contre les nations" (1)). Au-delà, s'esquisse une seconde couronne, lointaine, mal connue, neutre, celle des autres habitants du globe (notamment, les gens des "Iles").

Canaan, la terre promise, n'en est pas pour autant clairement délimitée. Un texte de la Genèse 1'étire "du fleuve d'Egypte au fleuve d'Euphrate". Plus couramment, les textes bibliques la situent entre "Dan et Bersabée" (du pied de l'Hermon à la frange du Négeb) seIon l'axe nord-sud, et, dans le sens Est-Ouest, "de la Mer à la Mer" (c'est à dire du Jourdain et de la mer Morte, d'un côté, à la Méditerranée, de 1 'autre) (2).

Cette imprécision ou ces divergences renvoient au contenu de l'espace. Il n'a ni les caractēristiques d'une "région", au sens traditionnel de ce terme, ni ceux d'un véritable Etat national. Il est le support de la population issue de Jacob.

La terre de Canaan, scindée, on le sait, en deux royaumes depuis le Xème siècle, constitue l'élément fort de la perception de leur environnement par les Juifs. Elle n'en demeure pas pour autant uniforme. A très grande échelle - celle de la maison, du champ, de la ville et tout particulièrement de "La Ville", Jérusalem - la saisie est vive. Au niveau micron-régional ou méso-régional, la représentation apparaît plus volontariste ou plus symbolique. Volontariste en ce sens que les articulations internes du territoire relèvent d'un partage réfléchi entre les douze tribus. Symbolique, voire stẻréotypée, en ce sens que l'évocation des lieux se limite à des clichés, le plus souvent ternes ("Le pays des montagnes", "le pays des collines", "Ainsi parla Yahvé aux ravins et aux vallées", etc...). Rien qui ressemble à ces descriptions moirées et chargées de suc comme savaient en donner les géographes de la traditions vidalienne (Il faut relire à cet égard le portrait que D.Rops brosse de la Palestine) (3).

Prof. J.-L. Piveteau, Institut de Géographie, Université de Fribourg, 1700 Fribourg. 
Une connexion majeure avec le processus millénaire de sédentarisation.

L'impression d'approximation et d'échelons, les uns appuyës, les autres estompës, en matière de description de l'espace, reflète la diversitẻ des êcrits vétéro-testamentaires le caractère instable des données politiques et la conviction qu'avait Israël de son élection par Dieu; mais aussi, profonde et puissante, une connexion avec le lent processus de sédentarisation qu'achèvent de vivre les descendants d'Isaac (c'est Isaac qui, le premier dans son ethnie, "fit des semailles et récolte le centuple") (4).

L'attentat de Caīn contre Abel ne conserve, à l'époque qui nous occupe, que sa connotation morale. Il a perdu sa notation immédiate de conflit opposant des cultivateurs durs à des pasteurs manquant de pâturages. Au VIème siècle, le peuple israélite se compose de petits citadins et surtout de laboureurs. Il a acquis des réflexes ruraux. Rien, d'étonnant, aussi, à ce que le niveau des "pays" (au sens de Gallois) fasse dēfaut. La texture de 1 'espace devait très peu à ses villes. Non qu'elles aient manqué. Un semis de grosses bourgades ponctuait le territoire. Dans une imprécation qui se veut accablante, Jérémie s'écrie: "aussi nombreuses que sont tes villes sont tes dieux!" (5). Mais il s'agissait d'un réseau élémentaire de modestes centres fortifiés, rentiers du sol, ayant pour rôle de servir de refuge aux campagnards à chaque rezzou venu du Sud ou du Nord. On songe au monde agraire européen du haut Moyen-Age.

Toutes les traces de la période nomade n'ont pas totalement disparu, loin de là. Il en subsiste dans les structures sociales. Il en surgit dans les références culturelles. Le langage use indifféremment d'images pastorales ou agricoles. ("Ma tente est détruite, mes cordes sont coupēes ... personne pour tendre mes toiles" (6); et: "Mon bien aimé avait une vigne sur le coteau fertile; il la bêcha, il l'épierra, il y planta les raisin vermeil" (7).) Mais l'essentiel est ici: Canaan n'a pas le caractère c'un espace national, au sens moderne du terme, parce que la population qui l'habite se sent bien plus une communauté de "race" ou, mieux, de filiation, qu'une communauté de volonté. La Palestine, au VIème siècle, c'est la terre d'un peuple fidèle à son origine tribale.

L'absence d'analyse positive de l'articulation interne du territoire ( au profit d'un découpage normatif) tient à la même raison. Les subdivisions que mentionnent le livre des Nombres et celui d'Ezéchiel expriment un lotissement géant de Canaan. On identifie le sol à la personnalité du groupe qui l'occupe et non pas aux empreintes, au demeurant modestes, que celui-ci laisse dans le paysage.

UNE RELATION ACTIVE AVEC L'ESPACE, TENDUE ENTRE L'OFFENSIVE ET LA DEFENSIVE.

Un peu plus spécifique déjà, apparaît la dualité des relations qu'entretient Israël avec l'espace. Elle manifeste un esprit de frontière et un esprit de résistance.

Esprit de frontière et esprit de résistance.

L'attitude conquërante remonte loin dans le temps. Elle atteint un paroxysme entre le XIIIème siècle, au moment de la longue marche vers Canaan et de la main-mise brutale sur "sa terre grasse, ses maisons regorgeant de tous biens, ses citernes déjà creusées, ses vignes, ses oliviers, ses arbres fruitiers à profusion" (8). Mais elle connaît, depuis, bien des poussées. Ellle se réveille notamment après 1 'exil, avec des accents dominateurs ou simplement pionniers. "Elargis l'espace de ta tente, prescrit Yahvé, déploie largement les toiles qui t'abritent, allonge tes cordages ... car à droite et à gauche tu vas éclater, ta race va déposséder des nations et repeupler des villes abandonnées". (9) "Je rassemblerai les enfants d'Israël ... ils bâtiront des maisons et planteront des vignes" (10). Et Dieu ajoute, à l'adresse de ceux qui avaient réussi à échapper à la déportation du Babylonien: "contre eux $j$ 'enverrai l'épée, la famine et la peste jusqu'à ce qu'ils aient disparu du sol que j'avais donné à leurs près" (11).

On assiste au VIème sičle à l'amorce d'une mutation. Israël se voit appelé à un grand dessein d'apostolat mondial: "Je fais de toi la lumière des nations pour que mon salut atteigne jusqu'aux extrémités de la terre" (12). L'esprit de conquête, selon cette voie, faibli.t dans ses convoitises palestiniennes, mais pour prendre une coloration d'impērialisme religieux.

Le pays est aussi objet de défensive. Depuis la scission du royaume de Salomon et, très récemment, les victoires mutilantes d'Assourbanipal et de Nabuchodonosor, le peuple hébreu fait 1 'expérience crucifiante de 7 'éclatement et de la déportation. L'espace perd de son identité dès lors qu'une fraction de "ceux à qui il a èté donné" ne s'y trouve plus. La résistance à la dispersion (la "diaspora") conditionne l'existence du territoire.

Il y a également un front intērieur, plus spécifiquement religieux: celui du refus des contacts débilitants, du rejet des syncrētismes ambiguis. Yahvé apparait à beaucoup comme le Dieu de la traversée du désert, contemporain des temps rudes de la vie nomade. Sédentarisés, devenus gens de campagnes et de villes, en contact avec 
des populations plus raffinées, les descendants de Jacob se mettraient volontiers au goūt polythëiste du jour. Mais la foi transmise 1 'emporte et avec elle des implications territoriales, qui, seules, nous concernent ici. La hantise d'un amoindrissement de la transcendance de Dieu débouche sur des attitudes ségrégatives: de ghetto chaque fois qu'on est en position de faiblesse; d'apartheid, lorsqu'on se trouve en position de force. L'image mentale de la Terre sainte comporte un combat permanent contre la pollution spirituelle. La Bible, sans complaisance, parle de prostitution.

Une connexion majeure avec la conjoncture socio-politique.

Les causes de cette dynamique contrastée (d'immixion et de rejet) sont, on s'en doute, diverses et interférentes. Une d'entre elles, cependant, a des connexions avec les deux types de comportements. Elle explique, semble$t-i l$, une large part des faits.

Depuis le dêpart d'Ur en Chaldée, l'histoire des descendants d'Abraham - autant que celle d'autres peuples, mais plus que celle de bien des peuples - est mue par des forces attractives et répulsives. Alternées ou simultanées. Plus souvent simultanees, mais selon des dosages variables entre "pull" et "push". Deux exemples. La pression d'envahisseurs venus du Nord n'est assurēment pas étrangère au grand trek d'Abraham. Mais il parait peu douteux, et indépendamment de toute position de foi, que le premier des Patriarches, a, fondamentalement, senti un appel intērieur à un dépassement de lui-même. A l'inverse, la sortie d'Egypte appartient à la catégorie des expulsions. Mais un petit groupe d'hommes, Moïse en tête, a su donner à ce qui était avant tout une émigration contrainte, le sens d'un projet spatial: s'installer en Terre promise.

Mal placé entre les grands empires mésopotamien et égytien d'une part, et sur le seul passage entre l'Euphrate et le Nil d'autre part, installé par droit de conquête sur une terre déjà solidement occupée, jamais totalement soumise, et convoitée de l'Est comme de l'Ouest par d'autres petites "nations"; mais en même temps tenaillé par la faim de terres et des ambitions à la mesure de son courage, le peuple hébreu a dû, sans trêve, attaquer et se dēfendre.

Les évènements du VIème siècle n'ont pu qu'aviver ces deux pulsions. Le retour en Palestine après l'édit de Cyrus (538) fait penser aux émigrés regagnant la France après le retour des Bourbons. Les rapatriés de Babylone doivent se tailler une place et ils ne doutent pas de la justice de leur cause. En contrepoint de cet esprit de reconquête, et chez les mêmes, surgit un esprit de résistance. Il consiste à refuser l'offre de concours des Samaritains pour la reconstruction du Temple; et à répudier leurs femmes si elles sont d'origine étrangère. Il s'agit de rester purs et durs. La conservation de l'espace est à ce prix.

\section{UNE INTERPRETATION RELIGIEUSE DES RAPPORTS} AVEC L'ESPACE.

C'est dans l'interprétations qu'ils donnent de leurs rapports avec l'espace que les hébreux se singularisent véritablement.

L'espace occupe une place essentielle dans
la conception religieuse d'Israël

Sans doute le monothéisme constitue-t-il le point fondamental de la foi juive: un monothéisme transcendant (Dieu est indicible, on sait seulement qu' "Il est") et personnel (Dieu tēmoigne à l'égard de la descendance de Jacob d'une sollicitude de tous les instants, vibrante et ombrageuse); un monothéisme hanté par. les ombres du "déviationnisme" (les séductions des religions ambiantes s'exercent, constantes, insidieuses, à tous les niveaux de la hiërachie sociale).

Mais dans cette relation profonde et vulnérable avec Yahvé, l'espace occupe une place singulière. Il tient lieu de signe, il joue le rôle de test. Il symbolise et sanctionne l'alliance particulière (le "testament") que Dieu a conclue avec une poignée d'hommes, "son" peuple: "Ecoutemoi, ô ma nation ... tu comptes beaucoup à mes yeux, tu as du prix et je t'aime... Vois, je t'ai gravée sur les paumes de mes mains" (13). A Abraham, Dieu avait expliqué qu' "Il avait exploré un pays", "un pays qui ruisselait de lait et de miel", et qu'il "donnerait ce pays" à la postérité du patriarche. Cette terre, solennellement promise, n'a rien d'abstrait: c'est Canaan.

A la source de cette tradition religieuse, il y eut d'abord, très vraisemblablement, le souci de galvaniser des hommes que décourageaient des combats sans cesse à reprendre et dont l'objectif n'était autre que d'élargir un espace vital chichement mesuré; et aussi, très vite, le besoin de théoriser cette appropriation de terres. Il y eut probablement, de façon croissante, la nécessité d'affirmer l'identité socio-politique très réelle du peuple hébreu par une emprise sur un sol défini, dans un monde où tout ce qui avait de la puissance s'appuyait sur une paysannerie solidement implantée et sur des villes (L'Egypte, Assour, Babylone, Tyr ...).

Les effets de cette conception de l'espace insérée dans la foi.

Oubliant, au fil des siècles, ses enracinements historiques, ou majorant la force des aspirations à l'absolu qui ont donné un sens aux faits matériels, l'interprétation religieuse acquiert un dynamisme partiellement autonome et pèse alors sur le comportement des hommes. En ce qui 
touche à la façon de vivre l'espace, cela conduit à une contradiction saisissante.

D'une part, et sous réserve d'inventaire, le peuple juif se montre, au VIème siècle, plus attaché à son espace que ne le sont, au leur, les autres peuples.

La polarisation sur Canaan (réduite alors à la portion congrue) atteint une manière de paroxysme. La Judée (100.000 habitants peuit-être) prend visage d'un concentré de Terre promise; et au coeur de la Judée, Jérusalem, la ville sainte, possède désormais dans l'ordre de la centralité urbaine un attribut que les agglomérations actuelles du plus haut niveau tertiaire ignorent: une valeur sacramentelle. Tout le peuple "s'y rassemble comme un seul homme" (14) à temps rêguliers.

La rentrée en possession de cet espace est tenue par les Israélites pour une preuve de leur élection et pour un signe de la puissance de leur Dieu, autant que pour une condition, bienvenue, de sécurité matérielle. L'exil, lui, avait été compris comme une disgrâce consēcutive aux infidélitēs commises à l'égard de la Loi. Il avait fait prendre conscience de l'aliénation au mal. Le peuple, lorsqu'il dévie, se condamne à se voir arracher de son sol. Il est en mesure ainsi, en quelque sorte, de fabriquer l'éclatement de son espace. Il ne peut, en revanche, fabriquer son espace. Cela, Yahvé se le réserve.

Par ailleurs, dans cette Palestine témoin tangible d'une affection êlective, et comme telle suscitant une passion terrienne qui mord au coeur, les Hébreux se trouvent moins enracinés que bien d'autres peuples dans leurs terres respectives. En toute logique.

Ils voient en elle, en effet, le don gratuit de Dieu, beaucoup plus que le résultat d'un façonnement progressif par le labeur accumulé de gēnērations successives. Yahvé, par la bouche de ses prophètes, ne marchande pas les mises au point. C'est lui qui a dispersé les enfants d'Israël; c'est lui qui les a, depuis, rassemblés; c'est lui qui redonne la terre, et toute construction procède de lui: "Les nations qui survivront autour de vous sauront que c'est moi, Yahvé, qui ai rebâti ce qui était démoli". (15)

L'espace saint, en second lieu, a pour mission de manifester la gloire de Dieu et non de servir l'appétit de croissance et de puissance de chacun de ses habitants. "La terre m'appartient et vous n'êtes pour moi que des hôtes" (16). Cette terre elle-même chômera, un an sur sept, dans un esprit d'allégeance. Et ce qu'elle produit sera offert en hommage au Seigneur, dans son Temple, à Jérusalem. Le drainage de la plus-value foncière est assuré par le réseau des prêtres et la richesse converge vers Sion. Ce prélèvement, non négligeable dans une économie agricole climatiqement vulnérable, répond également à une autre intention: éviter que 1 'homme ne prenne goût au sol et à ses fruits au point d'en perdre de vue l'essentiel. La terre, comme Eve, est tentatrice.

L'espace donné par Dieu demeura, enfin, de toute manière une possession précaire, puisque (le Peuple êlu le sait; ses prophètes, en tout cas, ne se font pas faute de le lui rappeler) toute infraction l'égard de la Loi risque de provoquer la colère et le dēsaveu de Dieu. ("Ils ont souillé leur territoire par leur conduite et leurs oeuvres ... alors j'ai déversé ma fureur sur eux ... je les ai dissēminés" (17).) Cette relativisation de la possession est démobilisante, souvent, à court terme: que l'on songe au défaitisme de Jérémie. L'idée que le retour vers Canaan vérifie l'hypothèse de l'Alliance a elle, au contraire, et à long terme, des vertus hautements stimulantes.

\section{CONCLUSION}

L'espace tel qu'il est vëcu par le peuple hébreu en cette seconde moitié du VIème siècle, combine donc:

- des traits qui s'inscrivent dans le temps long d'une sédentarisation graduelle, mais présentement révolue.

- des traits qui s'inscrivent dans le temps court d'une conjoncture politique mouvementēe et, tout récemment encore, traumatisante.

des traits, enfin et surtout, qui procèdent de la représentation que se fait ce peuple de ses relations avec son Dieu: celle d'un pacte dont la pierre de touche est l'emprise concrète qu'il peut, lui, l'héritier d'Israël, exercer sur un sol défini.

Le cas des Hébreux ne paraît, analytiquement, qu'inégalement généralisable à d'autres lieux et d'autres temps: il est donc, globalement, original.

Rēférences des passages de la Bible citēs.

$1 \quad$ Ez. $25-30, \mathrm{Jr} .46-51$

2 Nb. 34.1, Ez. $47-48$

3 D.Rops, Histoire Sainte, Paris, 1943, p.139.

4 Gn. 26.12

$5 \mathrm{Jr} .11 .13$

6 Jr. 10.20

7 Is. 5.1

8 Ne. $9.7-8$

9 Is. 54.2

10 Ez. 28.25

11 Jr. 24.5-7

12 Is. 49.6

13 Is. $51.4,43.4,49.16$

14 Esd. 3.1-2

15 Ez. 36.34

16 LV. 25.2-3

17 Ez. 36.17 